\title{
Evidence based medicine: concerns of a clinical neurologist
}

\author{
L R Caplan
}

The latest medical buzz phrase is evidence based. This term has become a shibboleth, a sacrosanct icon almost like motherhood. Who could possibly be against basing decisions on evidence? It is difficult to think of a polite term for decisions not based on evidence. The almost religious zeal for cloaking all decisions under the banner of evidence based conceals the real problem-that is, what is the evidence? Does it apply to the individual patient being treated? What is the context in which the evidence was gathered and it will be applied?

When historians look back on the last quarter of the 20th century, they will emphasise four developments: (1) dramatic improvement in diagnostic technology, especially brain related, (2) major advances in genetics and molecular biology, (3) computer facilitation of data bases and reference resources, and (4) focus on treatment and therapeutic trials. Greater interest in treatment is a natural consequence of improved diagnostic capabilities. Although therapeutic trials were conducted in the 1950 s, during the past two decades the methodology and the statistical planning and analysis of trials have developed a more sound scientific basis.

Recently, there has been a determined effort to integrate results of therapeutic trials into medical care. This integration is the strongest type of "evidence base." Delivery of therapeutic data from randomised trials, on line, to practising physicians has only recently become a reality. Doctors now have rapid access to diagnostic and therapeutic data bases using portable or office based computers. This capability brings scientific medicine into doctors' offices, and hospitals quickly and cheaply.

Marriage of the therapeutic and computer eras has led to the proposition that treatment should be based on data from therapeutic trials. Treatment based on trials is naturally preferred over treatment not based on trials. Most treatment reviews now contain wording that reflects the scientific quality of evidence supporting therapeutic recommendations. Table 1 lists weighting of evidence, and the grading of therapeutic recommendations used in two recent publications. ${ }^{12}$

This also threatens to become the managed care era. Managed care is rampant in the United States and is spreading rapidly. The trend is likely to spread to Europe and elsewhere in the world; economics seem more important than quality of care. Some managers warmly embrace treatments based only on trial data. Realising that little that doctors now do is based firmly on trial results, managers save money if they pay only for scientifically proved treatments. Payers generally look favourably on protocols that dictate to physicians accepted treatments based only on trial evidence. "Evidence based medicine" is thus a two edged sword, a utopia if accomplishable, but a potential managed-care sword. I argue herein that: (1) trials and meta-analyses have important limitations which make it unlikely that they will become the basis for most daily bedside medical decisions; and (2) whether a treatment is "evidence based" depends both on the quality of the evidence and the clinical context in which the evidence is applied.

\section{Randomised therapeutic trials placed in clinical perspective}

Trials have important theoretical and practical limitations. They are expensive, time consuming, and require enormous resources. To provide statistically valid results, randomised trials must contain large numbers of patients with enough end points for analysis. Sufficient end points must be obtained in a relatively short period. The condition studied must either be acute and cause adverse end points or rapid improvement within a short time. Chronic conditions must be severe enough to cause clear end points within 1 to 5 years of follow up. Many medical and neurological conditions are very difficult to study in trials. Patients who are too ill, too old, too young, female and "of childbearing age," incapable of giving informed consent, too complex, or too full of coexisting illnesses are often excluded in trials.

The major theoretical limitation of trials is the issue of numbers versus specificity. For trials to yield statistically valid and important results, they must include many patientsnumbers. For the results to be useful to practising physicians, the data must be specifically applicable to individual patients who have the condition studied. To include 
enough patients, the condition studied must be common and usually multiple physicians at multiple centres must be used. One doctor or centre has too few patients or would take unacceptably long to accrue the number of patients needed. To achieve numbers, a "lumping" strategy must predominate over "splitting". For example, to study the effectiveness of a treatment to prevent embolism in patients with mitral valve prolapse, a study would not be able to obtain enough patients with mitral valve prolapse, mitral regurgitation, and mitral valve fibrinoid degeneration who had prior brain or systemic emboli and congestive heart failure even though this group is at highest risk and would be most likely to respond to prophylaxis. The study would have to include all patients with mitral valve prolapse to accrue enough patients.

The sample size needed will increase if the projected effectiveness of the treatment (the percentage reduction in adverse outcomes) is low $(10 \%-15 \%)$; several treatments will be studied; the follow up period is short, many patients will be lost to follow up, withdraw, or become noncompliant; the anticipated outcome event rate is low; and a high power of protection from type I and type II errors is desired. ${ }^{3}$ As an example of the extraordinary numbers of patients sometimes needed, the authors of a meta-analysis of randomised controlled trials of agents that decrease platelet aggregation for secondary prevention of stroke calculated that 13000 patients would be needed to detect with $90 \%$ power, an observed reduction in end points of $15 \%$ with aspirin. ${ }^{4}$

The greater the numbers of patients needed, the more pressure there is to adopt a lumping strategy. The more a study lumps diverse subgroups, the more general are the results and applicability to specific patients declines. For practising physicians, treatment must be very specific. Physicians are faced with individual patients for whom they must make therapeutic decisions. To be useful, trial results must help physicians treat individual patients in given situations. In the free world, no physician is faced with treating thousands of individual patients with the same treatment irrespective of their individual characteristics but that is exactly the situation in trials. Randomised trials and metaanalyses estimate the effect of a particular treatment in a large group of patients. The results of that treatment are compared with patients given another treatment or placebo.

Subgroups can be managed either by prospective stratification - that is, by randomising patients using predetermined criteria (for example, sex, race, age) to ensure that subgroups will be relatively equally represented in the different treatment groups - or by analysing the treatment results by subgroup determinants that have been prospectively defined.

\section{Utility of trial information}

Trials should ask clinically relevant questions that are practically answerable. An example of one such useful trial is the extracranial/intracranial (EC/IC) bypass study. ${ }^{5}$ When this study was conceived, neurosurgeons throughout the world operated on patients with occlusive anterior circulation disease by surgically creating shunts from the superficial temporal artery to middle cerebral artery (MCA) branches. The study was large; 1377 patients were more than enough to ensure statistically valid results for the full group analysis. The mix of patients was representative of patients operated on in the community. Both transient ischaemic attacks (TIAs) and non-disabling strokes were included, and the operative procedure was performed about 6 weeks after stroke, the prevailing community practice. The chosen end point-new stroke related neurological deficits - was valid and readily ascertainable. The study showed that the surgical procedure was no better, and sometimes less effective than medical therapy. ${ }^{5}$ Especially convincing and important were the subgroup analyses. Groups of patients with lesions at various sites (internal carotid artery (ICA), origin, ICA siphon, MCA, different severities of occlusion, and shunts with different projected flow volumes were studied. The full group results were also valid for each subgroup. This trial convincingly showed that the procedure as it was then performed was less effective than medical treatment. The results led to a dramatic change in performance of the procedure. The study did not include metabolic and blood flow data but this was not then feasible. Some other randomised trials are much less useful to practising physicians. In this category are the stroke prevention trials of drugs that decrease platelet aggregation. Some studies showed in full group analyses a benefit for aspirin, aspirin combined with sulfinpyrazone or dipyridamole, ticlopidine, and clopidogrel. ${ }^{4-22}$ Unfortunately, the patient mix treated with antiplatelet aggregants or placebo was probably not representative of patients in the community presenting with transient ischaemic attacks (TIAs) or minor strokes. In none of these studies was classification of the nature and severity of the causative vascular and cardiac lesions required for entry. Patients with lesions thought favourable for carotid surgery were often operated on and were ineligible. Patients with "surgical" lesions deemed unfit for surgery-and patients unfit for angiography-were included in medical treatment groups. Some patients with detected cardiac sources of emboli were not entered. No systematic evaluation for carotid artery or cardiac disease was mandated. Subgroup analysis was only by sex and tempo of ischaemia (TIA, reversible ischaemic neurological deficits, minor stroke). The tempo of ischaemia does not predict the nature, severity, or locale of causative vascular lesions. ${ }^{23}{ }^{24}$ Because cardiac studies were not required, the groups also must have contained patients with brain embolism of cardiac origin as the cause of their ischaemia. A meta-analysis of randomised control trials of antiplatelet agents in the secondary prevention of stroke ${ }^{4}$ found "for aspirin compared with placebo a non-significant reduction in stroke of $15 \%$. . a trend in reduction of stroke for any regimen containing aspirin." ${ }^{4}$ The results of these studies are difficult for physicians to apply to individual patients with stroke with identified stroke mechanisms - for example, stenosis of the MCA or cardiogenic embolism. In defence of the studies cited, the technology available now-high quality duplex ultrasound scans, pulsed and continuous wave Doppler ultrasound, transcranial Doppler ultrasound, CT angiography, MR angiography, and echocardiography-were not widely available when the studies were designed. To recruit enough patients, the decision was made not to require angiography for entry (the numbers versus specificity issue). The result is that the data, despite enormous expense, are not very useful for physicians treating patients with the conditions studied in the trials. Future trials of antiplatelet aggregants should be conceived differently and have sufficient subgroup data related to the presence and severity of vascular lesions to be meaningful to practising physicians.

\section{Practical problems facing trials}

The logistics of performing randomised therapeutic trials can also be problematic.

FUNDING

Trials are big operations. Multiple centres require many physicians and clerical staff. Computer hardware and software and statistical expertise are needed to record, manage, interpret, and analyze data. The personnel and equipment 
are very costly. Money for funding comes either from governmental or private sources. Much time and effort is expended in writing grants and considerable pilot data are required. Government funding is becoming scarcer. Alternatively, private industry may fund grants if their products are studied. Potential problems arise from involvement of private enterprises that have much to gain and much to lose, depending on trial results. Many companies want to dictate trial methodology and/or play a part in analysis and publication of the results as a condition for financing studies. Companies have bailed out of studies depending on company finances and goals. Many worthwhile trials go unfunded.

ENTRY CHARACTERISTICS, EXCLUSIONS, AND CONSENT Inclusion and exclusion criteria are designed so that patients entered will be "pure breed" and will be able to be followed up until study completion. The plethora of exclusions often makes it difficult to recruit enough patients to meet sample size requirements. Estimates of the number of patients a centre thinks that it will recruit are usually at least two or three times more than they actually do enter once the trial begins. Trials studying the effectiveness of warfarin in preventing stroke in patients with atrial fibrillation encountered this recruiting problem. Physicians participating in these studies screened 15-25 patients with atrial fibrillation to obtain each eligible patient. In some trials patients who are eligible under the inclusion/ exclusion rules of a trial are not entered by physicians, who think that a particular patient needs the treatment and should not be randomised.

Eligible patients are not always easy to enroll in trials. Many patients decline because they do not want to be "guinea pigs" and view trials as something "others" do, especially "charity cases". Some patients are put off by the acknowledged lack of a scientific basis for treatment and cannot accept that a "flip of the coin" decides treatment. Some are disturbed that neither they nor their physicians will know what treatment they receive. Especially disconcerting is the prospect that they may receive a placebo. They think that their problem is serious and warrants active treatment. With time and patience some of these patients can be enrolled, but with much effort. Alas, some who have enrolled will be dissuaded later by their children, Aunt Tilly, or an all knowing friend, and will drop out. The medical profession must spend more time with public relations and the media to help public education about trials.

FORMS AND UNIFORMITY OF EVALUATION

To document that all procedures have been followed and all necessary examinations and evaluations have been performed, most studies require mountains of paper. Completion of forms takes time. Nurses, study coordinators, and clerks can accurately record demographic and some laboratory data, but often the filling out of important clinical data is delegated to very junior investigators and not senior clinicians. The validity of the clinical data is thus jeopardised. The results are only valid if the data are reliable and accurate. Senior experienced clinicians should have seen all patients and personally reviewed the forms to ensure accuracy but this is often not done.

NEED FOR QUANTIFICATION

To compare the effectiveness of different treatments, outcomes must be measured and quantified. This is simple if events such as death or new stroke are used, but are all strokes equal? In non-fatal diseases, other criteria-for example, degree of neurological deficit, disability, or other "objective" measures are used. Especially in neurology, severity and disability scores are problematic. How can aphasia be compared with diplopia, ataxia, facial numbness, and limb weakness? How are weights assigned to various abnormalities? For some patients, a hemianopia that makes reading difficult but does not effect daily living poses no major problem but to a physician, editor, or surveyor the same deficit is devastating.

\section{Making decisions about treatment in the clinic:} applying the results of trials to patient care

Having explored some theoretical and practical trial limitations, let us now turn to a real patient and see how information from trials is applied in the clinic.

A 63 year old Chinese woman has had three attacks during the past 4 weeks. During the first attack her right hand and face tingled for 5 minutes. During the second attack, which occurred 10 days later, her right arm and hand went weak and numb and she could not move her fingers. She also could not think of words and the words she used were sometimes incorrect. Her husband had difficulty understanding her. She could walk without difficulty during the attack, which lasted about 25 minutes. Eleven days later she had the third attack during which she momentarily could not speak and noticed numbness and tingling in her right face and hand and she limped when she walked. This attack lasted only about 2 minutes. She had been told of high blood pressure 5 years ago but only took the medicine prescribed when she had a headache or felt poorly. She came sporadically for follow up visits and liberally used chinese herbs and other remedies.

Blood pressure was 155/90. Pulse 80 and regular. General and neurological examinations were normal except for slight cardiomegaly, and brisker refexes in her right arm and leg. Ultrasound examinations of her neck and head showed high blood flow velocities in the left MCA. Brain MRI showed a small parietal lobe cortical infarct and MRA showed severe stenosis of the left MCA. The right MCA had a minor degree of stenosis. The neck arteries were normal. Electrocardiography showed slight left ventricular enlargement. A transoesophageal echocardiogram was normal.

The diagnosis in this patient is clear; she has been having TIAs in the left cerebral hemisphere caused by severe stenosis of the left MCA. In addition she has a small "silent" infarct in the same vascular territory although she gave no history of any persisting neurological deficit. Studies of patients from Boston's Chinatown area, ${ }^{25}$ where this patient lives, and Hong Kong, ${ }^{26}$ showed that intracranial occlusive disease is common among Asians, especially women. Moreover, I knew from past experience ${ }^{25}$ and discussions with colleagues and observations during visits to Hong Kong, and other Asian countries (Japan, Singapore, Thailand) that the clinical course and response to anticoagulants and possibly other medications was probably different in Asians compared with white people.

I searched trial data relevant to choosing treatment in this patient. I first looked at the results of trials of patients with TIAs and minor strokes. ${ }^{4-22}$ The data were clear that aspirin, aspirin with another antiplatelet aggregant, ticlopidine, and clopidogrel had been shown in trials with low $\alpha$ and $\beta$ errors and with convincing meta-analysis data to be somewhat effective in patients with TIAs and minor strokes. Ticlopidine was slightly more effective than aspirin but had more potential toxicity. ${ }^{19}{ }^{20}$ Clopidogrel was as effective as ticlopidine and had less toxicity. ${ }^{21}$ Aspirin when combined with modified release dipyridamole was more effective than either aspirin or dipyridamole alone in preventing stroke. ${ }^{22}$ The evidence could be classified as grade A according to published criteria. ${ }^{12}$ I explored these trials and meta-analyses further for their relevance to my patient. None of the trials included data on the vascular 
occlusive lesions that caused the brain ischaemia. There were no data in any of these analyses on patients with MCA stenosis or on intracranial artery stenosis.

I next searched reports of patients with MCA disease, ${ }^{25-35}$ but these series (except for the EC/IC bypass trial ${ }^{5}$ ) were descriptive and contained no prospective or randomised treatment data. These reports contained some retrospective data that white patients with MCA disease fared better on warfarin-type anticoagulants. These data would be a low grade $\mathrm{C}$ at best. However, these retrospective data applied to white patients. Is it reasonable to extrapolate the data to Chinese people? The distribution of vascular disease is different in Asians and white people, and the pathology of the vascular disease in the MCAs may differ from ICA atherostenosis, a disease predominantly of white men, in whom it has been more extensively studied. Rescanning the published reports indicated that Asians did not do as well on anticoagulant treatment as white patients and the clinical syndromes differed. Asians had more deep striatocapsular infarcts whereas white patients had more surface infarcts, and white people had more TIAs whereas Asians and black people had more strokes. Disease of the MCA was probably different in Asians compared with white people. Asians and black people are posited to have more disease of the vascular media and infarcts due to hypoperfusion distal to stenosis whereas white people might have more intimal disease with luminal white and red thrombi causing distal intra-arterial embolisation. ${ }^{28} \mathrm{I}$ could not extrapolate the results of medicinal treatments, anecdotal as they were, from treatment of white patients to treatment of my Chinese woman patient. I could conclude that EC/IC bypass was ineffective in patients with MCA stenosis. $^{5}$

I next looked for randomised trials of anticoagulant treatment in stroke and TIA patients. Unfortunately, these studies were mostly old, and none defined the causative vascular occlusive lesions. A Swedish study performed during the 1980s compared aspirin and anticoagulant drugs in patients with carotid territory TIAs. ${ }^{36}$ There was no difference in the frequency of recurrent ischaemia between the patients treated with aspirin and those treated with anticoagulant. The vascular lesions in this study were not defined and there were likely no Asians studied. ${ }^{36} \mathrm{~A}$ recent study analyzed outcomes in patients with intracranial occlusive disease treated with warfarin-type anticoagulants and platelet antiaggregants. ${ }^{37}$ In this study, which was a retrospective review rather than a randomised trial, patients with intracranial stenosis, most commonly of the MCA, fared better on warfarin than on platelet antiaggregants. This study included 97 white patients, 42 black patients, and 12 "others"; 83 patients had intracranial anterior circulation vascular occlusive lesions including 35 MCA stem and four MCA branch lesions. ${ }^{37}$ The report did not analyze results by race; nor was it stated how many of the "others" ( $8 \%$ of the total) were Asians. Because the centre in which I had worked entered some Chinese patients, I knew that the study included Asians. The report analyzed outcome in anterior and posterior circulation groups but not by individual vessels. Clinicians searching the literature by computer might try to contact the first author to find more specific analyses of the MCA cases and the results in Asians. If I did not know the first author, I would contact him at the address given in the published report only to find that he had moved-thus significantly delaying contact and increasing the time the patient would remain untreated. The data favouring anticoagulant treatment in this study would rate at best a low grade $\mathrm{C}$ by published evidence based criteria (table 1). ${ }^{12}$

Using these data, my own anecdotal experience, and information about the pathology and pathophysiology of brain ischaemia in general and in Asians, I decided that warfarin anticoagulation was probably the most effective medical treatment. However, there were specific problems with this treatment in this patient. She had hypertension and did not take hypertensive medications reliably. She used herbs and some chinese foods and herbs contain substances known to have anticoagulant properties. Furthermore, she had been non-compliant in the past and not reliable in coming to clinic for follow up. She spoke only Chinese so that detailed explanations and discussions about anticoagulant treatment might be suboptimal. An alternative treatment was angioplasty of the MCA lesion. Little has been published about the results of angioplasty in intracranial occlusive disease. ${ }^{38-42}$ Most reports are of angioplasty of extracranial carotid and vertebral artery lesions. I reviewed our own experience at the New England Medical Center with patients with MCA stenosis treated with angioplasty. ${ }^{40}$ Although most patients did well, impairment of flow in the lenticulostriate arteries was a problem in patients in whom the angioplasty involved the portion of the MCA from which the perforators originated. Angioplasty would entail a moderately high risk of stroke in this patient who had no important clinical deficit and whose lesion involved the proximal MCA including the lenticulostriate origins. I would consider this treatment only if medical therapy failed.

I decided, despite the relative contraindications, to try anticoagulant treatment because in my experience and that in the literature, the likelihood of stroke without treatment was high. I explained the treatment in detail on several occasions to try to ensure compliance. Did I make the correct decision? Time will tell how she does. We received little guidance from trials in making this spcific decision, so that our choice could not be considered evidence based. Would a managed care protocol for this patient with cerebrovascular disease dictate the use of platelet antiaggregants, as their use is evidence based by criteria in table 1? Would care managers decline payment for vascular tests as the nature, location, and severity of vascular lesions have not been unequivocally shown to effect response to treatment? Vascular lesions have not been shown to not effect treatment; this issue has simply not been studied formally.

Perhaps I could have saved time by using the Cochrane data base. I also do not want to seem completely negative about trial data. Studies convincingly show that some general medical measures-for example, treatment of hypertension-reduce the risk of cardiovascular and cerebrovascular events. I do mean to emphasise that trial data did not help with specific treatment recomendations for her specific cerebrovascular situation.

\section{Conclusions}

Practical and theoretical limitations of randomised therapeutic trials make it highly unlikely that trials will be able to provide relevant evidence for many, if not most, therapeutic decisions that physicians make daily. Evidence from trials is often general, but the evidence must be applied in the context of therapeutic decisions that relate to individual patients with specific problems. Insufficient specific information from the general results of trials means that many therapeutic decisions are in fact not really evidence based. Managed care and managers may try inappropriately to limit or pay only for treatments that they consider evidence based. They may not apply evidence based to the context of specific therapeutic decisions.

(1) Randomised therapeutic trials have not been performed on many therapeutic decisions that physicians make daily.

Arnold Relman, the former editor of the New England Fournal of Medicine wrote in an editorial that: "Randomised 
controlled trials are not the only way to obtain useful clinical information; sometimes they may not be necessary to evaluate the outcome of therapeutic interventions. Much depends on the question being asked and the nature of the condition being studied." ${ }^{43}$

Ethical issues limit consideration of many presently accepted but formally untested treatments. ${ }^{44}$ Could we now ethically randomise patients with appendicitis to no surgery, or patients with pneumococcal pneumonia or streptoccoccal pharyngitis to non-penicillin treatment? The ethics of some trials, especially in third world countries, continue to be debated. ${ }^{45} 46$

(2) Nuances, limitations, and imperfections of the methodology and results of many trials sometimes are apparent only to experienced clinicians who work directly within the subspecialty domains of those trials.

An example of the need for clinical context information not discussed in the initial trial report is the issue of timing of surgery in the EC/IC bypass trial. ${ }^{5}$ When this trial was planned and carried out, surgeons customarily waited 4 to 6 weeks after strokes before performing bypass surgery. Surgeons had been alerted by reports of brain haemorrhages after carotid endarterectomy. The haemorrhages were thought to be related to reperfusion of ischaemic brain shortly after infarction. Surgeons waited to perform shunts for fear of creating reperfusion haemorrhages. The delay in surgery of 4 to 6 weeks after strokes in the trial was the prevailing current practice. Subsequent studies have shown that the commonest time for progression and reinfarction is during the first hours and days after initial ischaemia. The risk of further stroke diminishes with time. Few strokes occur after a month. The EC/IC investigators found that stroke incidence after surgery performed 6 weeks after infarction was low but was not as low as in medically treated patients. ${ }^{5}$ The issue of the timing of surgery was not discussed in the initial report as it followed standard practice at that time. This nuance would not be evident to physicians who were not stroke specialists.

(3) The results of many randomised trials are impossible to apply directly to individual patients. The term evidence based must be used cautiously when applied to a particular circumstance if that circumstance has not been specifically studied.

Information from trials must be weighted according to the context of specific treatment decisions. Conducting trials is different from caring for sick patients. In trials, the same treatments are given to all eligible patients depending only on randomisation. Departure from the specified treatment makes the results difficult to interpret. In the clinic, doctors treat individual patients. George Thibault said it well:

"We then need to decide which approach in our large therapeutic armamentarium will be most appropriate in a particular patient, with a particular stage of disease and particular coexisting conditions, and at a particular age. Even when randomised clinical trials have been performed (which is true for only a small number of clinical problems), they will often not answer this question specifically for the patient sitting in front of us in the office or lying in the hospital bed." 47

Courts continue to wrestle with the issue of the applicability of general laws and precedents from prior individual cases to the case being considered. Data from other sources are only relevant as evidence when they directly apply to the legal case being considered. Shouldn't the medical profession emulate the legal profession when it come to evidence-for evidence is really a "legal" term. Although there may be general evidence that aspirin has some effectiveness against brain ischaemia, there may be no evidence that it is effective or ineffective for a given vascular disorder unless it has been specifically tested for that disorder. Even when it has been tested for that disorder, coexisting factors in that particular patient often complicate the decision about whether or not to use a particular agent in a particular patient at a particular time. Therapeutic decisions are often complex and require experienced physicians. Treatment decisions for individual patients cannot be made by protocols, rules, or computer searches. Evidence from trials, past experience, and intimate detailed knowledge of the patient, the diseases, and the wishes and desires of all concerned are required to make difficult therapeutic decisions. Nonphysicians should not be allowed to control this decision making process.

(4) Meta-analysis and systematic evidence based reviews ${ }^{48-50}$ are useful but are limited by the quality of the individual trials analyzed and reviewed.

The whole can only be the sum of its parts. Poor data, amalgamated or analyzed even by the best statistical methodology, are still inferior data. Meta-analysis of available trials of warfarin-type anticoagulants in the treatment of brain ischaemia (excluding specific trials for prophylaxis in patients with atrial fibrillation) is of little use as none of the original trials yielded useful data. None required diagnosis or evaluation of the cause of ischaemia. Attempts to homogenise and summate disparate heterogeneous protocols and methodologies is a problem.

We need more and better randomised therapeutic trials designed by clinicians to answer clinically relevant therapeutic dilemnas. We need more critical reviews of trials and therapeutic dilemmas by experienced senior clinicians. Inexpert reviews by young academics often miss nuances and often lack clinical perspective and experience.

For the reasons cited, the panacea and saviour for medical therapeutics is not, and will not be randomised trials or evidence based reviews or meta-analyses. Some envisage that the bulk of medical care will be delivered by primary care physicians who will spend much time at the computer reviewing evidence bases to guide therapeutic decisions. I suggest that also desperately needed is more time spent by specialists at the bedside and in the clinic finding out exactly what is wrong with each patient and getting to know each patient and their thoughts, fears, biases, and wishes. Therapeutic decisions are made with, by, and for complex people. They cannot be readily homogenised without losing the essence of what being a doctor is all about.

Department of Neurology, Harvard Medical School, Dana 779, Beth

Israel Deaconess Medical Center, 330 Brookline Ave, Boston 02215, MA, USA

lcaplan@caregroup.harvard.edu

1 Cook DJ, Guyatt GH, Laupacis A, et al. Rules of evidence and clinical recommendations on the use of antithrombotic agents. Chest 1992;102(suppl 4):305S-11S.

2 Biller J, Feinberg WM, Castaldo JE, et al. Guidelines for carotid endarterectomy. A statement of healthcare professionals from a special writing group of the Stroke Council, American Heart Association and the American of the Stroke Council, American Heart Associan of Neurology. Stroke 1998;29:554-62.

3 Meinert CL. Clinical trials: design, conduct, and analysis. New York, Oxford Meinert CL. Clinical tri
University Press, 1986.

4 Sze P, Reitman D, Pincus M, et al. Antiplatelet agents in the secondary prevention of stroke: meta-analysis of the randomized control trials. Stroke 1988;19:436-42.

5 EC/IC Bypass Study Group. Failure of extracranial-intracranial arterial bypass to reduce the risk of ischemic stroke: results of an international randomized trial. N Engl f Med 1985;313:191-200.

6 Fields WS, LeMak NA, Frankowski RF, et al. Controlled trial of aspirin in cerebral ischemia. Stroke 1977;8:301-16.

7 The Canadian Cooperative Study Group: a randomized trial of aspirin and sulfinpyrazone in threatened stroke. N Engl f Med 1978;299:53-9.

8 The American-Canadian Cooperative Study Group. Persantine-aspirin trial in cerebral ischemia. Part 1l. Endpoint results. Stroke 1985;16:406-15. 
9 Bousser MG, Schwege E, Haguenau M, et al. "AICLA" controlled trial of spirin and dipyridamole in the secondary prevention of atherothrombotic aspirin and dipyridamole in the secondary

10 European Stroke Prevention Study Group. ESPS: principal end points. Lancet 1987;ii:1351-4.

11 Britton M, Helmers C, Samuelsson K. High dose acetylsalicylic acid after cerebral infarction: a Swedish co-operative study. Stroke 1987;18:325-34.

12 UK-TIA Study Group. The United Kingdom transient ischaemic attack IUK-TIA) aspirin trial: final results. F Neurol Neurosurg Psychiatry 1991 ;54: 1044-54.

13 The Dutch TIA Trial Study Group. A comparison of two doses of aspirin $30 \mathrm{mg} v 283 \mathrm{mg}$ a day) in patients after a transient ischemic attack or minor ischemic stroke. N Engl f Med 1991;325:1261-6.

14 The SALT Collaborative Group. Swedish aspirin low-dose trial (SALT) of $75 \mathrm{mg}$ aspirin as secondary prophylaxis after cerebrovascular ischaemic events Lancet 1991;338:1345-9.

15 Antiplatelet Trialists' Collaboration. Secondary prevention of vascular disease by prolonged antiplatelet treatment. BMF 1988;296:320-31.

16 Antiplatelet Trialists' Collaboration. Collaborative overview of randomized trials of antiplatelet treatment. I. Prevention of death, myocardial infarction and stroke by prolonged antiplatelet therapy in various categories of patients. BMF 1994;308:81-106.

17 Candelise L, Landi G, Perrone P, et al. A randomized trial of aspirin and sulfinpyrazone in patients with TIA. Stroke 1982;13:175-9.

18 Matias-Guiu J, Davalos A, Pico M, et al. Low dose acetylsalicylic acid (ASA) plus dipyridamole versus dipyridamole alone in the prevention of stroke in patients with reversible ischemic attacks. Acta Neurol Scand 1987;76:41321.

19 Gent M, Blakely JA, Easton JD, et al. The Canadian American ticlopidine study (CATS) in thromboembolic stroke. Lancet 1989;i:1215-20.

20 Hass WK, Easton JD, Adams HP Jr, et al. A randomized trial comparing ticlcopidine hydrochloride with aspirin for the prevention of stroke in highrisk patients. N Engl F Med 1989;321:501-7.

21 CAPRIE Steering Committee. A randomised, blinded, trial of clopidogrel versus aspirin in patients at risk of ischaemic events (CAPRIE). Lancet 1996;348:1329-39.

22 Diener HC, Cunha L, Forbes C, et al. European stroke prevention study 2. Dipyridamole and acetylsalicylic acid in the secondary prevention of stroke. 7 Neurol Sci 1996;143:1-13.

23 Caplan LR. Are terms such as completed stroke, or RIND of continued usefulness? Stroke 1983;14:431-3.

24 Caplan LR. TIAs: we need to return to the question, "What is wrong with Mr Jones?" Neurology 1988;38:791-3.

25 Feldmann E, Daneault N, Kwan E, et al. Chinese-white differences in the distribution of occlusive cerebrovascular disease. Neurology 1990;40:15415.

26 Huang YN, Gao S, Li SW, et al. Vascular lesions in Chinese patients with transient ischemic attacks. Neurology 1997;48:524-5.

27 Caplan LR, Babikian V, Helgason C, et al. Occlusive disease of the middle cerebral artery. Neurology 1985;35:975-82.

28 Lhermitte F, Gautier JC, Derouesne C. Nature of occlusion of the middle cerebral artery. Neurology 1970;20:82-8.
29 Bogousslavsky J, Barnett HJM, Fox AJ, et al for the EC/IC bypass Study Group. Atherosclerotic disease of the middle cerebral artery Stroke Group. Atheroscler.

30 Naritomi H, Sawada T, Kuriyama Y, et al. Effect of chronic middle cerebral artery stenosis on the local cerebral hemodynamics. Stroke 1985;16:21419.

31 Saito I, Segawa H, Shiokawa Y, et al. Middle cerebral artery occlusion: correlation of computed tomography and angiography with clinical outcome. Stroke 1987;18:863-8.

32 Hinton R, Mohr JP, Ackerman R, et al. Symptomatic middle cerebral artery stenosis. Ann Neurol 1979;5:152-7.

33 Corston RN, Kendall BE, Marshall J. Prognosis in middle cerebral artery stenosis Stroke 1984;15:237-41.

34 Moulin DE, Lo R, Chiang J, et al. Prognosis in middle cerebral artery occlusion. Stroke 1985;16:282-4.

35 Feldmeyer JJ, Merendaz C, Regli F. Stenosis symptomatiques de l'artere cerebral moyenne. Rev Neurol 1983;139:725-36.

36 Garde A, Samuelsson K, Fahlgren $\mathrm{H}$, et al. Treatment after transient ischemic attacks: a comparison between anticoagulant drug and inhibition of platelet aggregation. Stroke 1983;14:677-81.

37 Chimowitz MI, Kokkinos J, Strong J, et al. The warfarin-aspirin symptomatic intracranial disease study. Neurology 1995;45:1488-93.

38 Higashida RT, Halbach W, Tsai FY, et al. Interventional neurovascular technique for cerebral revascularization in the treatment of stroke. AfR Am $\mathcal{F}$ Roentgenol 1994:163:793-800.

39 Clark WM, Barnwell SL, Young LM, et al. Safety and efficacy of angioplasty for intracranial atherosclerotic stenosis [abstract]. Stroke 1994;25:273.

40 Takis C, Kwan E, Pessin MS, et al. Intracranial angioplasty,experience and complcations. AfNR Am F Neuroradiol 1997;18:1661-8.

41 Nakano S, Yokogami K, Ohta $\mathrm{H}$, et al. Direct percutaneous transluminal angioplasty for acute middle cerebral artery occlusion. AfNR Am f Neuroradiol 1998;19:767-72

42 Suh DC, Sung K-B, Cho YS, et al. Transluminal angioplasty for middle cerebral artery stenosis in patients with acute ischemic stroke. AfNR Am f Neuroradiol 1999;20:553-8.

43 Relman A. What have we learned about the treatment of idiopathic membranous nephropathy with steroids. N Engl f Med 1989;320:248-9.

44 Alves WA, Macciocchi SN. Ethical considerations in clinical neuroscience. Current concepts in neuroclinical trials. Stroke 1996:27:1903-9.

45 Angell $M$. The ethics of clinical research in the third world. $N$ Engl f Med 1997;337:847-9.

46 Varmus H, Satcher D. Ethical complexities of conducting research in developing countries. N Engl f Med 1997;337:1003-5.

47 Thibault GE. Clinical problem solving: too old for what? $N$ Engl f Med 1993;328:946-50.

48 Cook DJ, Mulrow CD, Haynes RB. Systematic reviews: synthesis of best evidence for clinical decisions. Ann Intern Med 1997;126:376-80

49 Mulrow CD, Cook DJ, Davidoff F. Systematic reviews: critical links in the great chain of evidence. Ann Intern Med 1997;126:389-91.

50 LeLorier J, Gregoire G, Benhaddad A, et al. Discrepancies between meta-analyses and subsequent large randomized, controlled trials. N Engl f Med 1997;337:536-42.

\section{EDITORIAL COMMENTARIES}

\section{Getting to the heart of dementia}

The two commonest causes of dementia are probably dementia of the Alzheimer type (DAT) and dementia with Lewy bodies (DLB), and although their pathology is relatively well characterised (albeit controversial in terms of diagnostic features) the clinical expression of these disorders can sometimes be difficult to distinguish. Typically DAT presents with deficits in episodic memory with language involvement whereas in DLB it is more hallucinations with a fluctuating dementia in the context of a parkinsonian syndrome. Distinguishing these two disorders, however, is not always straightforward and in this issue (pp 583-588) Yoshito et al $^{1}$ claim that such a distinction can be made using, rather surprisingly, a peripheral marker of sympathetic activity- $\left[{ }^{123} \mathrm{I}\right]$ MIBG myocardial scintigraphy.

MIBG is a physiological analogue of noradrenaline (norepinephrine) and is used to label sympathetic postganglionic nerve terminals and so has been most extensively used for diseases targeting the autonomic nervous system, including a range of parkinsonian plus disorders. ${ }^{23}$ Yoshida et al now report that myocardial $\left[{ }^{123} \mathrm{I}\right]$ MIBG is significantly reduced in patients with DLB irrespective of duration of disease or degree of autonomic failure, a situation that is not seen in DAT even when a degree of autonomic failure is documented. This therefore suggests that in both conditions there may be involvement of central autonomic pathways but that in DLB there is additional pathology involving peripheral sympathetic processes; an involvement that is not related to medication.

It is now established that some extrapyramidal diseases have pathology that can involve both the central and peripheral autonomic nervous system (see for example, Stoddard et $a l^{4}$ ). However, DLB has not consistently been shown to have any such pathology but this study clearly suggests that this is likely, although it produces no postmortem data to confirm the fact. Indeed the study would be greatly strengthened if the MIBG abnormalities could be correlated with a specific pathological process and the absence of postmortem confirmation of disease in the 
two patient groups also limits interpretation. The recruitment of consecutive patients, with its inherent variability, can be seen as a strength of the study, but it also creates problems due to the range of disease seen-for example, patients' mini mental state examination scores ranged from 4 to 26 . It is therefore not clear how confident one can be about the positive findings irrespective of disease duration or severity given the few patients at each data point. Indeed it could be argued that comparing only mildly affected patients may be more useful as it is this group that represent the greatest challenge both diagnostically and therapeutically - although the absence of effective long term therapies may even limit this potential at the present time.

In conclusion, this study highlights the possibility that neurodegenerative disorders targeting the cerebral cortex may be distinguished through their effects on sympathetic nerve endings in the heart. However, perhaps the most important message from this study is to highlight once more that neurodegenerative disorders display widespread pathology and that this needs to be accommodated in any theory of pathogenesis as well as novel therapeutic approaches.

R A BARKER

Department of Neurology and Cambridge Centre for Brain Repair, Addenbrooke's Hospital, Hills Road, Cambridge CB2 2QQ, UK

rab46@cus.cam.ac.uk
1 Yoshita M, Taki J, Yamada M. A clinical role for ${ }^{123}$ I MIBG myocardial scin- tigraphy in the distinction between dementia of the Alzheimer's type and dementia with lewy bodies. J Neurol Neurosurg Psychiatry 2001;71:583-8.
2 Yoshita M. Differentiation of idiopathic Parkinson's disease from striatoni- gral degeneration and progressive supranuclear palsy using iodine-123- metaiodobenzylguanidine myocardial scintigraphy. J Neurol Sci 1998;155: 60-7.
3 Druschky A, Hilz MJ, Platsch G, et al. Differentiation of Parkinson's disease and multiple system atrophy in early disease stages by means of I-123-MIBG-SPECT. J Neurol Sci 2000;175:3-12
4 Stoddard SL, Ahlskog JE, Kelly PJ et al. Decreased adrenal medullary catecholamines in adrenal transplanted parkinsonian patients compared to nephrectomy patients. Exp Neurol 1989;104:218-22.

\section{Videodynamic and sphincter motor unit potential analyses in Parkinson's disease and multiple system atrophy}

In the paper by Sakakibara et al (this volune, pp 600-606), ${ }^{1}$ the differences in findings of investigations of bladder function between patients with Parkinson's disease and multiple system atrophy are reported. The authors' new findings focus on the urethral sphincter mechanism, examining this functionally (using some of the newer urodynamic indices) and fluoroscopically. But the paper also provides a useful summary, reviewing as it does, previous work in this area, which has so far been mainly published in the urological literature. Although urinary symptoms can be troublesome in advanced Parkinson's disease they do not have the same prominence and severity as those seen in early multiple system atrophy, probably due to the multiple defects of neurological control of the bladder and sphincter function that develop in the initial stages of multiple system atrophy. Abnormalities of urethral sphincter innervation, both the external (striated) and intrinsic (bladder neck) are marked as a feature of multiple system atrophy but not Parkinson's disease, as has been shown in this study.

There has been a tendency up to now to include bladder symptoms as part of the "autonomic failure" which characterises multiple system atrophy, but the growing realisation that the entire system of neurological control of the bladder is selectively involved in its early stages, may lead to a new approach in understanding the evolution of this progressive and fatal neurodegenerative disease.

C J FOWLER

Department of Uro-Neurology, National Hospital for Neurology and Neurosurgery, Queen Square, London WC1N 3BG, UK

c.fowler@ion.ucl.ac.uk

1 Sakakibara R, Hattori T, Uchiyama T, et al. Videodynamic and sphincter motor unit potential analyses in Parkinson's disease and multiple system atrophy. $\mathcal{F}$ Neurol Neursurg Psychiatry 2001;71:600-6.

\section{Sphincter EMG in possible multiple system atrophy: to do or not to do?}

In multiple system atrophy (MSA) the motor neurons of sphincter muscles (Onuf's nucleus) degenerate earlier than those of limb muscles (Sung et $a l^{1}$ ). Such lower motor neuron loss is, in principle, accompanied by muscle pathology, which may be readily assessed by EMG methods, of which there are several. Although there is no doubt that expertise may be accumulated with any of the various methods, data comparing different techniques are only beginning to appear. Gilad et al (this issue, pp 596-99) report on results obtained by concentric needle EMG (CNEMG) single fibre EMG (SFEMG), and interference pattern (IP) analysis in a small group of patients with MSA. ${ }^{2}$ Their findings both confirm and contradict the commonly held views on sphincter EMG findings in MSA. As expected, some EMG abnormalities were found in all patients studied, but the reported changes in isolation (reduced 
recruitment pattern with a reduced number of active motor units during rest) are not specific for loss of lower motor neurons. Comparing patients with MSA and healthy controls, no significant differences in CNEMG and SFEMG parameters (which are known to correlate with reinnervation changes in muscle) were found. Thus, the "new" parameter may either be more sensitive in detecting early degeneration of Onuf's nucleus, or reflect upper motor neuron involvement. The failure of Gilad et $a l^{2}$ to detect significant "classic" EMG abnormalities cannot be due to insensitivity of their CNEMG analysis (although the "multi-motor unit potential (multi-MUP) technique" has problems with detection of unstable complex MUPs, held to be typical of MSA) because of their confirmatory SFEMG findings. Thus, their results can actually be interpreted as showing that many patients with "possible" or "probable" MSA have no degeneration of Onuf's nucleus! However, practically all previous studies have demonstrated obvious ("classic") EMG abnormalities! The dissenting voices in the literature do not question the presence of EMG abnormalities in MSA, but whether these are significantly different from those in other degenerative extrapyramidal diseases (the literature is ample and has recently been reviewed- see Vodušek ${ }^{3}$ ). The results of Gilad et $a l^{2}$ thus question the value of "classic" sphincter EMG more radically (however, based on results of only 11 patients). The "majority opinion" of studies of a total of well over 500 patients with MSA still supports the "expert opinion" that the finding of pathological spontaneous activity and/or significant MUP abnormalities in the sphincter muscle favours - in the appropriate clinical setting - the diagnosis of MSA. The "appropriate setting" includes caveats that the patient with parkinsonism should be in the first 5 years of the disease course, and that he or she has no other cause for sphincter denervation; EMG does not differentiate between MSA and progressive supranuclear palsy. The absence of EMG abnormalities, however - and this was appreciated even before the article by Gilad et $a l^{2}$-does not rule out MSA (see Vodušek ${ }^{3}$ ).

The techniques of EMG and the assessed parameters used up until now have varied, and thoroughly explored normative data from a large control group with a well standardised method of quantitative CNEMG have only recently been published-by Podnar et $a l,{ }^{4}$ and now by Gilad et al. The method ("multi-MUP") allows standardised quantitative assessment of a sizeable sample of motor unit potentials (MUPs) with little personal bias. With IP analysis there is even less personal bias, with Gilad et al stressing the informative value of this method in patients with MSA. It seems that the above mentioned methods should be the logical choice in future studies, which should clarify the time course of sphincter motor neuron degeneration in MSA, preferably combining methods demonstrating functional and structural changes.

D B VODUŠEK

Division of Neurology, University Medical Centre, Zaloška 7, 1525

Ljubljana, Slovenia

david.vodusek.@kcli.si

1 Sung J, Mastri A, Segal E. Pathology of Shy-Drager syndrome. J Neuropathol Exp Neurol 1979;49:353-68

2 Gilad R, Giladi N, Korczyn AD, et al. Quantitative anal sphincter EMG in multisystem atrophy and one hundred controls. J Neurol Neurosurg Psychiatry 2001;71:596-9.

3 Vodušek DB. Sphincter EMG and differential diagnosis of multiple system atrophy. Mov Dis $2001 ; 16: 600-7$.

4 Podnar S, Vodušek DB, Stålberg E. Standardization of anal sphincter electromyography: normative data. Clin Neurophysiol 2000;111:2200-7. 